\title{
乌ु
}

\section{Dark Matter Velocity Spectroscopy}

\author{
Eric G. Speckhard, ${ }^{1,2, *}$ Kenny C. Y. Ng, ${ }^{1,2, \dagger}$ John F. Beacom, ${ }^{1,2,3, \ddagger}$ and Ranjan Laha ${ }^{4, \S}$ \\ ${ }^{1}$ Center for Cosmology and AstroParticle Physics (CCAPP), Ohio State University, Columbus, Ohio 43210, USA \\ ${ }^{2}$ Department of Physics, Ohio State University, Columbus, Ohio 43210, USA \\ ${ }^{3}$ Department of Astronomy, Ohio State University, Columbus, Ohio 43210, USA \\ ${ }^{4}$ Kavli Institute for Particle Astrophysics and Cosmology (KIPAC), \\ Department of Physics, Stanford University, Stanford, California 94035, USA; \\ and SLAC National Accelerator Laboratory, Menlo Park, California 94025, USA
}

(Received 31 July 2015; published 19 January 2016)

\begin{abstract}
Dark matter decays or annihilations that produce linelike spectra may be smoking-gun signals. However, even such distinctive signatures can be mimicked by astrophysical or instrumental causes. We show that velocity spectroscopy - the measurement of energy shifts induced by relative motion of source and observer-can separate these three causes with minimal theoretical uncertainties. The principal obstacle has been energy resolution, but upcoming experiments will have the precision needed. As an example, we show that the imminent Astro-H mission can use Milky Way observations to separate possible causes of the $3.5-\mathrm{keV}$ line. We discuss other applications.
\end{abstract}

DOI: 10.1103/PhysRevLett.116.031301

Introduction.-What is the dark matter (DM)? Identification depends upon more than just observation of its bulk gravitational effects; distinct particle signatures are needed. Backgrounds make it difficult to pick out these signals, which are constrained to be faint. Among possible decay or annihilation signals, those with sharp spectral features, such as a line, are especially valuable.

Given that the stakes and difficulties are so profound, even such a "smoking-gun" signal may not be conclusive. A line could have other causes: astrophysical (baryonic) emission or detector backgrounds (or response effects). For example, the cause of the recently discovered $3.5-\mathrm{keV}$ line is disputed [1-13]. This problem is more general [14-23] and will surely arise again. We need better evidence than just a smoking gun-we need to see it in motion.

Premise and motivation.-We propose a general method for distinguishing the possible causes of a sharp spectral feature. Consider a line of unknown cause-dark matter, astrophysical, or detector-observed in the Milky Way (MW). Relative motion between source and observer leads to distinctive energy shifts as a function of line of sight (LOS) direction. Figure 1 illustrates this schematically. Because typical Galactic virial velocities are $\sim 10^{-3} c$, the Doppler shifts are only $\sim 0.1 \%$. Though exploiting such shifts is a standard astronomical technique (see, e.g., Refs. [24-29]), this is the first demonstration of their power for testing DM signals.

A potential target for DM velocity spectroscopy is the 3.5-keV line recently observed in MW, M31, and galaxy cluster spectra $[1,2,4]$. The line energy and flux can naturally be explained by sterile neutrino DM [30-40] (or other candidates [41-50]). However, the significance of the line is disputed $[3,5,6]$, and it has been argued that it can be explained by astrophysical emission $[7,9]$.
With present detectors, velocity spectroscopy of this line is impossible. Excitingly, the Soft X-ray Spectrometer (SXS) on Astro-H (launch date early 2016) has a goal energy resolution of $\sigma_{\mathrm{AH}}=1.7 \mathrm{eV}(4 \mathrm{eV}$ FWHM) [51,52], which is at the $0.1 \%$ scale. We show that if this goal resolution is achieved, together with a reasonable exposure, Astro-H can identify the cause of the $3.5-\mathrm{keV}$ line through precise measurements of the centroid energies in different

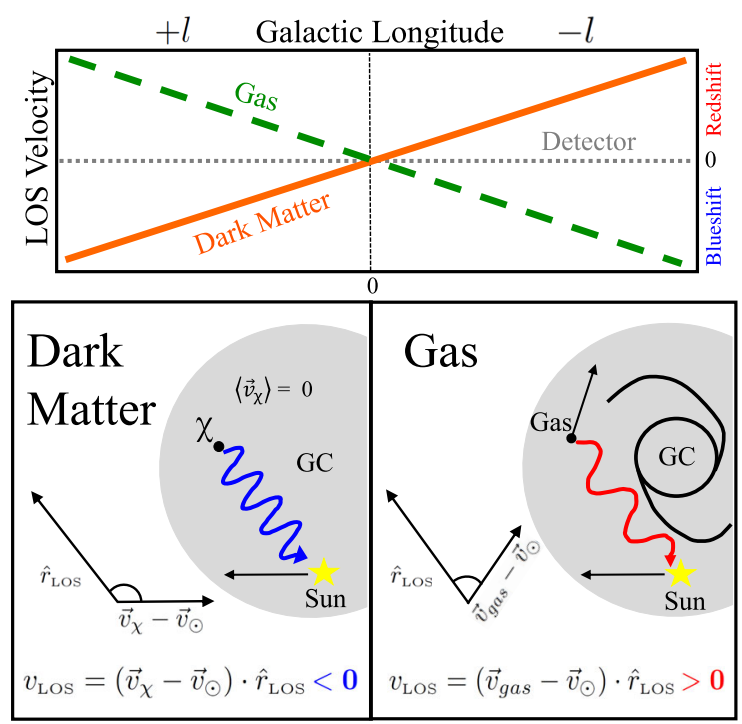

FIG. 1. Top: How DM, astrophysical, and detector lines shift with Galactic longitude is starkly different. Bottom: For DM signals at positive longitude, our motion through the nonrotating DM halo yields a negative LOS velocity and thus a blueshift. In contrast, for astrophysical lines (e.g., from gas), corotation in the disk leads to a positive LOS velocity and thus a redshift. These signs reverse at negative longitude. Detector lines have zero shift. 
directions. (More generally, this could be done in detectors with worse energy resolution but better statistics).

We emphasize that the applicability of DM velocity spectroscopy is much more general. The purpose of this Letter is to introduce a new concept to increase the power of DM searches and to spur innovation in detector design. We conclude by discussing several generalizations.

Usual DM decay signal.- The differential intensity (flux per solid angle) from DM with mass $m_{\chi}$ and lifetime $\tau=1 / \Gamma$, decaying within the MW, is

$$
\frac{d I(\psi, E)}{d E}=\frac{\Gamma}{4 \pi m_{\chi}} R_{\odot} \rho_{\odot} \mathcal{J}(\psi) \frac{d N(E)}{d E},
$$

where $R_{\odot} \simeq 8 \mathrm{kpc}$ and $\rho_{\odot} \simeq 0.4 \mathrm{GeV} \mathrm{cm}^{-3}$ [53-55] are the distance to the Galactic center (GC) and local DM density. (We neglect the cosmologically broadened extragalactic signal, which contributes negligibly in Astro-H's narrow energy bins.) $\mathcal{J}(\psi)$ is the dimensionless, astrophysical $J$-factor defined by the LOS integral

$$
\mathcal{J}(\psi) \equiv \frac{1}{R_{\odot} \rho_{\odot}} \int d s \rho_{\chi}(r[s, \psi]),
$$

where $\psi$ is the angle relative to the $\mathrm{GC}$ and is related to Galactic longitude and latitude via $\cos \psi=\cos l \cos b$. $d N(E) / d E$ is the photon spectrum.

The above treatment assumes that the astrophysical term $\mathcal{J}(\psi)$ and the photon spectrum $d N(E) / d E$ are separable. However, for detectors with energy resolution $\lesssim 0.1 \%$, this approximation is not valid because relative velocities between source and observer, and therefore the spectral shape, vary along the LOS.

Modified DM spectrum.-We first account for how the signal is broadened by DM velocity dispersion and, second, for how it is shifted due to bulk relative motion.

We take the DM halo of the MW to be spherically symmetric, in steady state, and to have no appreciable rotation. The last is expected from angular momentum conservation, as the baryons from the protohalo have collapsed significantly, while the DM has not; this is confirmed by simulations [56,57]. Thus, $\left\langle\vec{v}_{\chi}\right\rangle=0$.

DM particles do have nonzero velocity dispersion, determined by the total gravitational potential of the halo $[58,59]$. Assuming an isotropic velocity distribution $\left(\sigma_{v, r}=\sigma_{v, \phi}=\sigma_{v, \theta}\right.$, so the total dispersion is $\left.\sqrt{3} \sigma_{v, r}\right)$, the radial velocity dispersion of DM is [58]

$$
\sigma_{v, r}^{2}(r)=\frac{G}{\rho_{\chi}(r)} \int_{r}^{R_{\mathrm{vir}}} d r^{\prime} \rho_{\chi}\left(r^{\prime}\right) \frac{M_{\mathrm{tot}}\left(r^{\prime}\right)}{r^{\prime 2}},
$$

where $M_{\text {tot }}(r)$ is the total mass within a radius $r$. Typical values at $r \sim$ few kpc are $\sigma_{v, r} \simeq 125 \mathrm{~km} \mathrm{~s}^{-1}$.

To calculate $\sigma_{v, r}(r)$, we adopt the mass model of Ref. [60], which fits a contracted DM and three-component baryon mass profile to MW rotation curve data; for more details, see Supplemental Material [61]. The choice of mass model is not critical; kinematic results from other models agree within $O(10 \%)$ [53,62].

The spectrum from a point along the LOS is the convolution of the intrinsic spectrum with the DM velocity distribution at that point. We assume a Maxwellian velocity distribution throughout the halo, which, at each point, yields a Gaussian distribution of the LOS velocity component. The modified spectrum from each point is

$$
\frac{d \tilde{N}(E, r[s, \psi])}{d E}=\int d E^{\prime} \frac{d N\left(E^{\prime}\right)}{d E^{\prime}} G\left(E-E^{\prime} ; \sigma_{E^{\prime}}\right),
$$

where $G\left(E ; \sigma_{E}\right)$ is a Gaussian of width $\sigma_{E}=(E / c) \sigma_{v_{\text {LOS }}}$. Based upon observations of the LOS velocity distribution of MW halo stars reported in Ref. [63], we take $\sigma_{v_{\text {LOS }}}(r) \simeq \sigma_{v, r}(r)$, which implies $\sigma_{E}=(E / c) \sigma_{v, r}(r[s, \psi])$.

The line shift follows from the LOS velocity, $v_{\text {LOS }} \equiv\left(\left\langle\vec{v}_{\chi}\right\rangle-\vec{v}_{\odot}\right) \cdot \hat{r}_{\text {LOS }}$, where positive $v_{\text {LOS }}$ indicates receding motion. For $v_{\mathrm{LOS}} \ll c$, the resultant energy shift is $\delta E_{\mathrm{MW}} / E=-v_{\mathrm{LOS}} / c$.

The Sun follows a roughly circular orbit about the GC in the direction toward positive Galactic longitude at a speed $v_{\odot} \simeq 220 \mathrm{~km} \mathrm{~s}^{-1}$ [64]. (Recent work suggests $v_{\odot} \simeq$ $240 \mathrm{~km} \mathrm{~s}^{-1}[65,66]$, which would strengthen our results.) The spectrum is therefore shifted by $\delta E_{\mathrm{MW}}(l, b) / E=$ $+\left(v_{\odot} / c\right) \sin l \cos b$, which changes sign with $l$. We neglect the solar peculiar velocity as well as Earth and satellite motions, as the in-plane components of these are each $\sim 10 \mathrm{~km} \mathrm{~s}^{-1}$ [67-69]; even in combination, these are at most a $~ 10 \%$ effect, and they enter with distinctive time scales.

The final expression for the modified spectrum, including broadening and shifts, is therefore

$\frac{d \mathcal{J}}{d E}=\frac{1}{R_{\odot} \rho_{\odot}} \int d s \rho_{\chi}(r[s, \psi]) \frac{d \tilde{N}\left(E-\delta E_{\mathrm{MW}}, r[s, \psi]\right)}{d E}$,

so that Eq. (1) is altered by $\mathcal{J}(\psi) d N(E) / d E \rightarrow d \mathcal{J}(\psi, E) /$ $d E$. The observed signal, which is the convolution of $d \mathcal{J} / d E$ with the detector response, is nearly Gaussian and has an effective width $\sigma_{\text {eff }}$.

Modified astrophysical spectrum.-The details are slightly different for astrophysical lines.

The widths of astrophysical lines are primarily determined by the mass of the emitting atom and by the gas temperature; turbulent broadening is negligible [70]. For potassium at $T=2 \mathrm{keV}$, the intrinsic linewidth is $\sigma_{\text {gas }} \simeq 0.8 \mathrm{eV}$, comparable to Astro-H's goal resolution, $\sigma_{\mathrm{AH}} \simeq 1.7 \mathrm{eV}$. The intrinsic width is weakly sensitive to the gas temperature and mass $(\propto \sqrt{T / m})$; any reasonable values of $T$ and $m$ give similar results.

For the shift of an astrophysical signal, we must account for corotation within the MW disk. (While there is a nonrotating, gaseous halo at the outskirts of the MW, its temperature, $\sim 0.1 \mathrm{eV}[5,71-73]$, is far below the 
multi-keV temperatures needed to produce significant line emission near $3.5 \mathrm{keV}$ [1]). For simplicity, we assume all baryons follow circular orbits about the GC with speed $v_{\text {circ }}(r)=\sqrt{G M_{\text {tot }}(r) / r}$. With this circular speed and the hot gas distribution of Ref. [74], we compute the spectral shift by integrating the signal along the LOS with the contribution from each point weighted by the gas density. We call this fiducial model G2.

Because the spatial and speed distributions of MW x-ray gas are uncertain, we compare to models in Ref. [28] with smaller and larger line shifts. G1 is based on the distribution of free $e^{-}$[75] and the MW rotation curve [76]. G3 is based on the observed distribution of ${ }^{26} \mathrm{Al}$ gamma rays [28]. G1 and $\mathrm{G} 2$ are in good agreement with $\mathrm{MW} \mathrm{HI}$ and $\mathrm{CO}$ data $[24,25]$. Peak LOS velocities for G1, G2, and G3 are $\simeq 50$, 75 , and $250 \mathrm{~km} \mathrm{~s}^{-1}$.

Line flux detection.-One prerequisite to detecting a spectral shift is that the number of signal events be nonzero. Another is that the background fluctuations be small in comparison. Though Astro-H has a small field of view (FOV), its excellent energy resolution strongly suppresses backgrounds for a line signal, so that even a small number of signal events can be significant.

Viewing directions $l \simeq 10^{\circ}-40^{\circ}$ have advantages. First, the balance between decreasing signal flux and increasing energy shift at large $l$ is optimized. Second, theoretical uncertainties are minimized, as the DM density profile at $r \gtrsim$ few kpc is fixed by rotation curve data. Third, continuum astrophysical backgrounds are reduced; we reduce these further by going slightly off the Galactic plane, which minimally affects the DM signal.

The expected signal intensity is calculated from Eq. (1). For our DM example, this is

$$
\begin{aligned}
I(\psi)= & 1.2 \times 10^{-8} \mathrm{~cm}^{-2} \mathrm{~s}^{-1} \operatorname{arcmin}^{-2} \\
& \times\left(\frac{\sin ^{2} 2 \theta}{7 \times 10^{-11}}\right)\left(\frac{m_{\chi}}{7 \mathrm{keV}}\right)^{4}\left(\frac{\mathcal{J}(\psi)}{\mathcal{J}\left(l=20^{\circ},|b|=5^{\circ}\right)}\right),
\end{aligned}
$$

where we have integrated over energy in the line profile, calculated $\mathcal{J}\left(l=20^{\circ},|b|=5^{\circ}\right)=7.5$ using Ref. [60], and taken the DM parameters from Ref. [1]. For Astro-H, $\Omega_{\mathrm{FOV}}=9 \operatorname{arcmin}^{2}$ and (conservatively) $\mathrm{A}_{\mathrm{eff}}=200 \mathrm{~cm}^{2}$ [51,52], so the expected number of events is

$$
N_{s}(\psi) \simeq 43\left(\frac{\mathcal{J}(\psi)}{\mathcal{J}\left(l=20^{\circ},|b|=5^{\circ}\right)}\right)\left(\frac{t}{2 \mathrm{Ms}}\right) .
$$

This assumed exposure is large, but appropriate to the stakes (a potential discovery of DM) and the difficulties (the total exposure of XMM, Chandra, and Suzaku used in the $3.5-\mathrm{keV}$ analyses is $\gtrsim 40 \mathrm{Ms}[1,2,4,77,78])$. Furthermore, due to Astro-H's excellent energy resolution, all pointings in a substantial fraction of the sky will help test the $3.5-\mathrm{keV}$ line.
For continuum backgrounds, we consider only the contribution over the narrow energy range $\pm 2 \sigma_{\text {eff }}$ centered at $3.5 \mathrm{keV}$. (We do not need to include the tails of nearby astrophysical lines, as they will be well resolved, unlike in XMM). One component of the background is due to the isotropic cosmic x-ray background (CXB) [79-81]. We conservatively adopt the total $\mathrm{CXB}$ flux (unresolved+ resolved sources), $E d \Phi_{\mathrm{CXB}} / d E=9.2 \times 10^{-7}(E / \mathrm{keV})^{-0.4}$ $\mathrm{cm}^{-2} \mathrm{~s}^{-1} \operatorname{arcmin}^{-2}$ [81]. Another background, due to hot gas in the MW, varies strongly with direction [82]. Finally, there are detector backgrounds due to intrinsic and induced radioactivities as well as cosmic-ray interactions; their intensity is expected to be comparable to that of the CXB [83]. For $\psi\left(l=20^{\circ},|b|=5^{\circ}\right)$, backgrounds contribute $N_{b} \simeq 5.2+5.4+5.4=16$ events per $2 \mathrm{Ms}$ within the $\pm 2 \sigma_{\text {eff }} \simeq \pm 4.8 \mathrm{eV}$ band centered at $3.5 \mathrm{keV}$, compared to $N_{s} \simeq 41$.

We estimate the detection significance by the Poisson probability $P(n \geq 57 \mid \mu=16)$, which corresponds to a one-sided Gaussian probability $>7 \sigma$.

Line shift detection.-Detecting a line shift depends on how well the centroid of the line profile is determined. Backgrounds decrease the precision, but, as above, the energy resolution of Astro-H plays a critical role.

When backgrounds are absent, the uncertainty on the centroid is $\sigma_{\text {eff }} / \sqrt{N_{s}}$. When they are present, the uncertainty becomes $\delta E=C(R) \sigma_{\text {eff }} / \sqrt{N_{s}}$, where $C(R)$ is a correction factor and $R$ is defined by the background-tosignal ratio. We calculate the optimal $C(R)$ using the Cramer-Rao theorem [84-86]. For $\psi\left(l=20^{\circ},|b|=5^{\circ}\right)$, $C(R) \simeq 1.6$, so that the uncertainty in the LOS velocity is $\delta_{v_{\text {LOS }}} \simeq 50 \mathrm{~km} \mathrm{~s}^{-1}$.

Figure 2 shows the line profiles at $\psi\left(l=20^{\circ},|b|=5^{\circ}\right)$ for a $3.5-\mathrm{keV}$ emission line, due to either DM or gas. (A detector line would have zero shift.) These profiles show how the energy spectra are shifted due to relative motion as well as broadened due to intrinsic dispersion and detector resolution. We show the uncertainties on the centroids, which are separated from each other and from zero in a 2-Ms exposure. With the energy resolution of XMM [87] $\left(\sigma_{\mathrm{XMM}} \simeq 47 \mathrm{eV}\right.$ versus $\left.\sigma_{\mathrm{AH}} \simeq 1.7 \mathrm{eV}\right)$, the profiles are indistinguishable.

Figure 3 shows how the expected shifts vary with Galactic longitude, along with their uncertainties, assuming 2-Ms observations for each point. We show the DM signal uncertainties: for an astrophysical line of the same flux, the uncertainties are comparable because the effective widths are comparable $\left(\sigma_{\text {eff }}^{\text {gas }} \simeq 160 \mathrm{~km} \mathrm{~s}^{-1}, \sigma_{\text {eff }}^{\text {DM }} \simeq 200 \mathrm{~km} \mathrm{~s}^{-1}\right)$; see Fig. 2. For a detector line with zero intrinsic width, the effective width is $\sigma_{\text {eff }}^{\text {det }} \simeq 150 \mathrm{~km} \mathrm{~s}^{-1}$, approximately a factor of $\sqrt{2}$ less than $\sigma_{\text {eff }}^{\mathrm{DM}}$.

For each point in Fig. 3, it is easy to assess the probability that the expected DM signal could fluctuate to match that expected for an astrophysical or detector line, i.e., that a true DM signal could remain hidden. With 


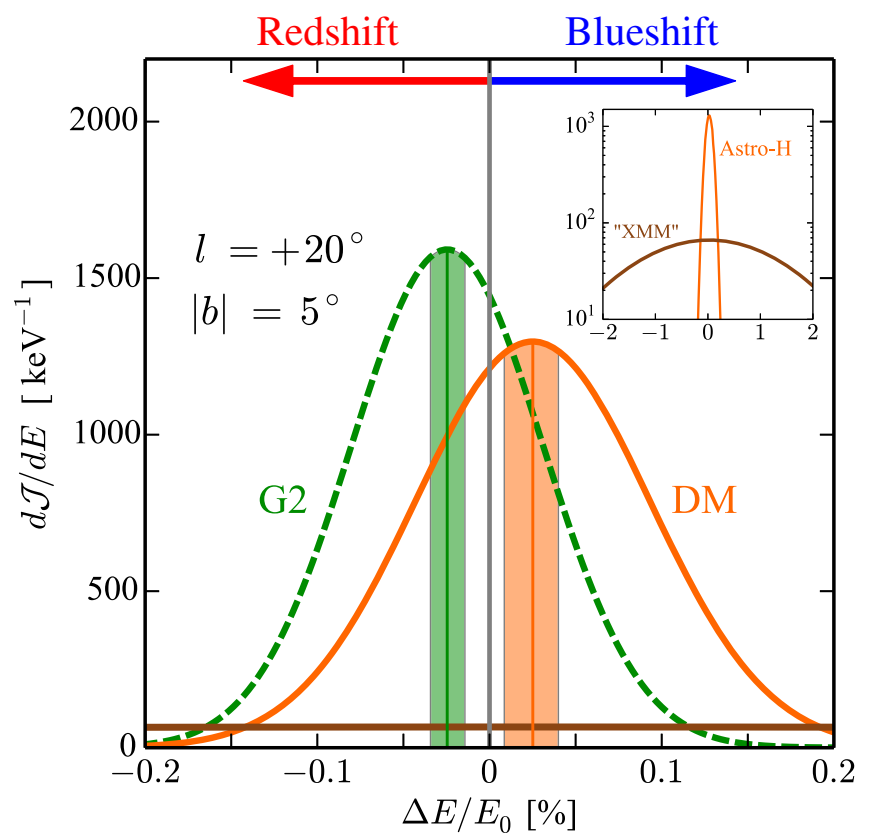

FIG. 2. Comparison of received spectra for DM and gas (G2). The emitted spectra are taken to have equal flux and to be centered at $3.5 \mathrm{keV}$ before velocity effects. The line profiles include velocity dispersion and shift effects, as well as the energy resolution of Astro-H. Vertical bands indicate the $1 \sigma$ centroid uncertainties after 2-Ms observations. For contrast, the brown line in the figure and the inset shows the same signal if Astro- $\mathrm{H}$ had the energy resolution of XMM.

two observations, at $l= \pm 20^{\circ}$, this scenario can be ruled out, relative to $\mathrm{G} 2$, at $\simeq 3.6 \sigma$. This establishes that this technique has interesting sensitivity. Once there are data, one can assess the probability that an astrophysical or detector line could mimic a DM signal (for the same flux, $\delta_{v_{\text {LOS }}}^{\mathrm{gas}} \simeq \delta_{v_{\mathrm{LOS}}}^{\mathrm{det}} \simeq \delta_{v_{\mathrm{LOS}}}^{\mathrm{DM}} / \sqrt{2}$ ).

If the energy resolution is worse than the design goal, e.g., $\sigma_{\mathrm{AH}} \simeq 2.1,2.5$, or $3 \mathrm{eV}$, then the line shift significance is $\simeq 3.0,2.4$, or $1.9 \sigma$ (the line flux significance is always $>5 \sigma$ ). This could be improved as $\sqrt{t}$ with more exposure (including nondedicated pointings). We have not included the systematic uncertainty due to detector gain calibration, for which the goal is $0.4 \mathrm{eV}$ [83]. This can be mitigated by comparing the energies of nearby astrophysical lines, especially at opposite longitudes.

Related searches.-Astro- $\mathrm{H}$ may be able to resolve the intrinsic width of a MW DM line. This would provide the first information on the large-scale DM velocity distribution, which is sensitive to DM particle properties [88] and to the presence of substructure [89,90] (see Supplemental Material [61]).

The 3.5-keV line has been detected in M31. Because of the relative motion between the Sun and M31, DM or astrophysical lines from M31 will have LOS shifts of $\simeq-300 \mathrm{~km} \mathrm{~s}^{-1}$ [91]. Provided the line energy has been measured precisely in other data, we estimate that this blueshift could be detected with $>5 \sigma$ significance, making this an attractive way to test detector causes. Because of

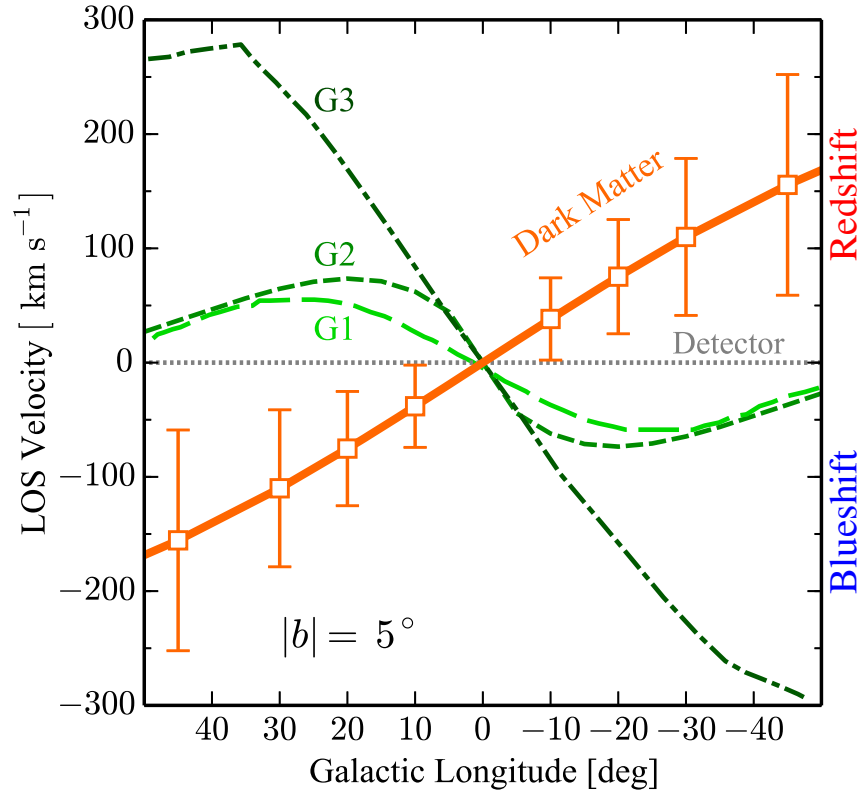

FIG. 3. LOS velocity for DM and various gas models (the realistic version of Fig. 1). Uncertainties are computed assuming 2-Ms Astro-H exposures on each point.

M31's rotation, astrophysical lines are separated from DM lines by $\pm 200 \mathrm{~km} \mathrm{~s}^{-1}$ around $\pm 1^{\circ}$, but, because the statistical uncertainties are large, they cannot be cleanly distinguished in $2 \mathrm{Ms}$; see Supplemental Material [61] and Refs. [92-98]. The Large Magellanic Cloud (LMC) [99] may also be an attractive target.

Cluster observations will also be important. For a single galaxy cluster, the cosmological recession velocity can be measured with Astro-H using atomic line emission from the intracluster gas. The large internal velocities of the cluster will broaden astrophysical and DM lines (differently, which is a potential discriminator $[1,83]$ ), making it somewhat more challenging to test line shifts (see above for how a larger line width can be compensated by larger statistics). For bright clusters, it may be possible to measure their velocities precisely, such that stacked observations increase the significance of the flux, as in Ref. [1], and also the velocity dependence of the centroid, allowing stronger tests of detector versus astrophysical or DM causes.

More speculatively, it may be possible to see the line in the extragalactic DM signal, if more astrophysical sources in the CXB are resolved, e.g., with eROSITA [100,101]. Furthermore, because we move at $\simeq 400 \mathrm{~km} \mathrm{~s}^{-1}$ with respect to the Comic Microwave Background (CMB), it may be possible to detect a dipole signature in DM line signal. Far-future observations may even detect a forest of sources in each LOS spectrum.

Conclusions. - Even for a supposedly smoking-gun signal, such as a line, it may be difficult to distinguish between $\mathrm{DM}$, astrophysical, or detector causes. We have shown that detectors with energy resolution $\lesssim 0.1 \%$ can break this 
degeneracy using velocity spectroscopy, which has minimal theoretical uncertainties. We emphasize that our main goal is to point out this new and robust method for testing DM signals, which can be applied to any sharp feature, such as an edge or box $[102,103]$.

To demonstrate the potential of this technique, we have shown that Astro-H will be able to test the origin of the $3.5-\mathrm{keV}$ line. In the future, other lines may be discovered. For lines at higher energy, the relative energy resolution of Astro-H improves. This unprecedented resolution will allow Astro-H to dramatically improve on existing sterile neutrino limits [104-122]. We encourage a dedicated study by the Astro-H Collaboration, once postlaunch parameters are known, to give definitive answers on DM sensitivity over their full energy range.

We are encouraged by the expected $0.1 \%$ resolution of Astro- $\mathrm{H}$ in the range $0.3-12 \mathrm{keV}$ and the demonstrated $0.1 \%$ resolution of INTEGRAL-SPI in the range $20 \mathrm{keV}-8 \mathrm{MeV}$ (including velocity spectroscopy of the $1.809-\mathrm{MeV}$ line from ${ }^{26} \mathrm{Al}$ [26-28]). Excitingly, the proposed x-ray mission ATHENA [123] and GeV gamma-ray mission HERD [124] have made achieving similar energy resolution a priority, which will improve existing limits [125-136]. We encourage other missions to pursue this aggressively.

We are grateful to Yoshiyuki Inoue, Matthew Kistler, Greg Madejski, Philipp Mertsch, Annika Peter, and Randall Smith for discussions. E. G. S. is supported by a Fowler Fellowship, K. C. Y. N. and J. F. B. by NSF Grant No. PHY-1404311 to J. F. B., and R. L. by KIPAC.

*speckhard.1@osu.edu †ng.199@osu.edu *beacom.7@osu.edu ${ }^{\S}$ rlaha@stanford.edu

[1] E. Bulbul, M. Markevitch, A. Foster, R. K. Smith, M. Loewenstein, and S. W. Randall, Astrophys. J.789, 13(2014).

[2] A. Boyarsky, O. Ruchayskiy, D. Iakubovskyi, and J. Franse, Phys. Rev. Lett. 113, 251301 (2014).

[3] S. Riemer-Sorensen, arXiv:1405.7943.

[4] A. Boyarsky, J. Franse, D. Iakubovskyi, and O. Ruchayskiy, Phys. Rev. Lett. 115, 161301 (2015).

[5] M.E. Anderson, E. Churazov, and J. N. Bregman, Mon. Not. R. Astron. Soc. 452, 3905 (2015).

[6] D. Malyshev, A. Neronov, and D. Eckert, Phys. Rev. D 90, 103506 (2014).

[7] T. E. Jeltema and S. Profumo, Mon. Not. R. Astron. Soc. 450, 2143 (2015).

[8] D. Iakubovskyi, arXiv:1410.2852.

[9] O. Urban, N. Werner, S. W. Allen, A. Simionescu, J. S. Kaastra, and L. E. Strigari, Mon. Not. R. Astron. Soc. 451, 2447 (2015).

[10] E. Carlson, T. Jeltema, and S. Profumo, J. Cosmol. Astropart. Phys. 02 (2015) 009.

[11] D. Iakubovskyi, Mon. Not. R. Astron. Soc. 453, 4097 (2015).
[12] D. Iakubovskyi, E. Bulbul, A. R. Foster, D. Savchenko, and V. Sadova, arXiv:1508.05186.

[13] A. Neronov and D. Malyshev, arXiv:1509.02758.

[14] M. Loewenstein and A. Kusenko, Astrophys. J. 714, 652 (2010).

[15] D. A. Prokhorov and J. Silk, Astrophys. J. 725, L131 (2010).

[16] C. Weniger, J. Cosmol. Astropart. Phys. 08 (2012) 007.

[17] D. P. Finkbeiner, M. Su, and C. Weniger, J. Cosmol. Astropart. Phys. 01 (2013) 029.

[18] M. Su and D. P. Finkbeiner, arXiv:1206.1616.

[19] F. Aharonian, D. Khangulyan, and D. Malyshev, Astron. Astrophys. 547, A114 (2012).

[20] E. Tempel, A. Hektor, and M. Raidal, J. Cosmol. Astropart. Phys. 09 (2012) 032; 11 (2012) A01.

[21] M. Ackermann et al. (Fermi-LAT Collaboration), Phys. Rev. D 88, 082002 (2013).

[22] C. Weniger, M. Su, D. P. Finkbeiner, T. Bringmann, and N. Mirabal, arXiv:1305.4710.

[23] M. Ackermann et al. (Fermi-LAT Collaboration), Phys. Rev. D 91, 122002 (2015).

[24] T. Dame, D. Hartmann, and P. Thaddeus, Astrophys. J. 547, 792 (2001).

[25] P. M. W. Kalberla and L. Dedes, Astron. Astrophys. 487, 951 (2008).

[26] K. Kretschmer, R. Diehl, and D. H. Hartmann, Astron. Astrophys. 412, L47 (2003).

[27] R. Diehl et al., Nature (London) 439, 45 (2006).

[28] K. Kretschmer, R. Diehl, M. Krause, A. Burkert, K. Fierlinger, O. Gerhard, J. Greiner, and W. Wang, Astron. Astrophys. 559, A99 (2013).

[29] S. E. Boggs et al., Science 348, 670 (2015).

[30] S. Dodelson and L. M. Widrow, Phys. Rev. Lett. 72, 17 (1994).

[31] X.-D. Shi and G. M. Fuller, Phys. Rev. Lett. 82, 2832 (1999).

[32] K. N. Abazajian, Phys. Rev. Lett. 112, 161303 (2014).

[33] K. Abazajian, G. M. Fuller, and M. Patel, Phys. Rev. D 64, 023501 (2001).

[34] M. Shaposhnikov and I. Tkachev, Phys. Lett. B 639, 414 (2006).

[35] A. Kusenko, Phys. Rev. Lett. 97, 241301 (2006).

[36] A. Merle, Int. J. Mod. Phys. D 22, 1330020 (2013).

[37] A. Merle, V. Niro, and D. Schmidt, J. Cosmol. Astropart. Phys. 03 (2014) 028.

[38] K. P. Modak, J. High Energy Phys. 03 (2015) 064.

[39] A. V. Patwardhan, G. M. Fuller, C. T. Kishimoto, and A. Kusenko, Phys. Rev. D 92, 103509 (2015).

[40] T. Venumadhav, F.-Y. Cyr-Racine, K. N. Abazajian, and C. M. Hirata, arXiv:1507.06655.

[41] D. P. Finkbeiner and N. Weiner, arXiv:1402.6671.

[42] T. Higaki, K. S. Jeong, and F. Takahashi, Phys. Lett. B 733, 25 (2014).

[43] H. M. Lee, S. C. Park, and W.-I. Park, Eur. Phys. J. C 74, 3062 (2014).

[44] M. Cicoli, J. P. Conlon, M. C. David Marsh, and M. Rummel, Phys. Rev. D 90, 023540 (2014).

[45] K.-Y. Choi and O. Seto, Phys. Lett. B 735, 92 (2014).

[46] M. T. Frandsen, F. Sannino, I. M. Shoemaker, and O. Svendsen, J. Cosmol. Astropart. Phys. 05 (2014) 033.

[47] S. P. Liew, J. Cosmol. Astropart. Phys. 05 (2014) 044. 
[48] E. Dudas, L. Heurtier, and Y. Mambrini, Phys. Rev. D 90, 035002 (2014).

[49] K. S. Babu and R. N. Mohapatra, Phys. Rev. D 89, 115011 (2014).

[50] S. B. Roland, B. Shakya, and J. D. Wells, arXiv:1506 .08195 [Phys. Rev. D (to be published)].

[51] T. Takahashi et al., Proc. SPIE Int. Soc. Opt. Eng. 8443, $84431 Z$ (2012).

[52] T. Takahashi et al., SPIE Conf. Ser. 9144, 914425 (2014).

[53] R. Catena and P. Ullio, J. Cosmol. Astropart. Phys. 08 (2010) 004.

[54] J. Bovy and S. Tremaine, Astrophys. J. 756, 89 (2012).

[55] M. Pato, F. Iocco, and G. Bertone, arXiv:1504.06324.

[56] J. S. Bullock, A. Dekel, T. S. Kolatt, A. V. Kravtsov, A. A. Klypin, C. Porciani, and J. R. Primack, Astrophys. J. 555, 240 (2001).

[57] M. Vitvitska, A. A. Klypin, A. V. Kravtsov, R. H. Wechsler, J. R. Primack, and J. S. Bullock, Astrophys. J. 581, 799 (2002).

[58] J. Binney and S. Tremaine, Galactic Dynamics (Princeton University Press, Princeton, 1987).

[59] B.E. Robertson and A. R. Zentner, Phys. Rev. D 79, 083525 (2009).

[60] A. Klypin, H. Zhao, and R. S. Somerville, Astrophys. J. 573, 597 (2002).

[61] See Supplemental Material at http://link.aps.org/ supplemental/10.1103/PhysRevLett.116.031301 for a description the mass models and velocity dispersion profile; the line of sight velocity dispersion; and the velocity spectroscopy of M31.

[62] P. J. McMillan, Mon. Not. R. Astron. Soc. 414, 2446 (2011).

[63] X. Xue et al. (SDSS Collaboration), Astrophys. J. 684, 1143 (2008).

[64] F. J. Kerr and D. Lynden-Bell, Mon. Not. R. Astron. Soc. 221, 1023 (1986).

[65] R. Schonrich, Mon. Not. R. Astron. Soc. 427, 274 (2012).

[66] J. Bovy et al., Astrophys. J. 759, 131 (2012).

[67] P. J. McMillan and J. J. Binney, Mon. Not. R. Astron. Soc. 402, 934 (2010).

[68] S. K. Lee, M. Lisanti, and B. R. Safdi, J. Cosmol. Astropart. Phys. 11 (2013) 033.

[69] A. H. Peter, V. Gluscevic, A. M. Green, B. J. Kavanagh, and S. K. Lee, Phys. Dark Univ. 5-6, 45 (2014).

[70] S. Redfield and J. L. Linsky, Astrophys. J. 613, 1004 (2004).

[71] X. Dai, M. E. Anderson, J. N. Bregman, and J. M. Miller, Astrophys. J. 755, 107 (2012).

[72] M. E. Anderson and J. N. Bregman, Astrophys. J. 737, 22 (2011).

[73] A. Gupta, S. Mathur, Y. Krongold, F. Nicastro, and M. Galeazzi, Astrophys. J. 756, L8 (2012).

[74] K. Ferriere, Astrophys. J. 497, 759 (1998).

[75] J. M. Cordes and T. Lazio, arXiv:astro-ph/0207156.

[76] Y. Sofue, M. Honma, and T. Omodaka, Publ. Astron. Soc. Jpn. 61, 227 (2009).

[77] T. Tamura, R. Iizuka, Y. Maeda, K. Mitsuda, and N. Y. Yamasaki, Publ. Astron. Soc. Jpn. 67, 23 (2015).

[78] N. Sekiya, N. Y. Yamasaki, and K. Mitsuda, Publ. Astron. Soc. Jpn. (to be published).
[79] A. Kushino, Y. Ishisaki, U. Morita, N. Y. Yamasaki, M. Ishida, T. Ohashi, and Y. Ueda, Publ. Astron. Soc. Jpn. 54, 327 (2002).

[80] A. De Luca and S. Molendi, Astron. Astrophys. 419, 837 (2004).

[81] R. C. Hickox and M. Markevitch, Astrophys. J. 645, 95 (2006).

[82] H. Uchiyama, M. Nobukawa, T. G. Tsuru, and K. Koyama, Publ. Astron. Soc. Jpn. 65, 19 (2013).

[83] T. Kitayama et al., arXiv:1412.1176.

[84] D. E. Muller, H. C. Hoyt, D. J. Klein, and J. W. M. DuMond, Phys. Rev. 88, 775 (1952).

[85] J. F. Beacom and P. Vogel, Phys. Rev. D 60, 033007 (1999).

[86] F. James, Statistical Methods in Experimental Physics (North-Holland Publishing, Amsterdam, 2006).

[87] M. J. Turner et al., Astron. Astrophys. 365, L27 (2001).

[88] M. Rocha, A. H. G. Peter, J. S. Bullock, M. Kaplinghat, S. Garrison-Kimmel, J. Onorbe, and L. A. Moustakas, Mon. Not. R. Astron. Soc. 430, 81 (2013).

[89] S. Ghigna, B. Moore, F. Governato, G. Lake, T. Quinn, and J. Stadel, Astrophys. J. 544, 616 (2000).

[90] M. Lisanti, D. N. Spergel, and P. Madau, Astrophys. J. 807, 14 (2015).

[91] L. Chemin, C. Carignan, and T. Foster, Astrophys. J. 705, 1395 (2009).

[92] R. Supper et al., Astron. Astrophys. 317, 328 (1997).

[93] R. Shirey et al., Astron. Astrophys. 365, L195 (2001).

[94] R. Bender et al., Astrophys. J. 631, 280 (2005).

[95] A. Bogdan and M. Gilfanov, Mon. Not. R. Astron. Soc. 388, 56 (2008).

[96] A. Tamm, E. Tempel, P. Tenjes, O. Tihhonova, and T. Tuvikene, Astron. Astrophys. 546, A4 (2012).

[97] M. Kaplinghat, R. E. Keeley, T. Linden, and H.-B. Yu, Phys. Rev. Lett. 113, 021302 (2014).

[98] P. A. R. Ade et al. (Planck Collaboration), arXiv:1502 .01589 .

[99] R. P. van der Marel, D. R. Alves, E. Hardy, and N. B. Suntzeff, Astron. J. 124, 2639 (2002).

[100] A. Merloni et al. (eROSITA Collaboration), arXiv: 1209.3114.

[101] F. Zandanel, C. Weniger, and S. Ando, J. Cosmol. Astropart. Phys. 09 (2015) 060.

[102] A. Ibarra, A. S. Lamperstorfer, S. L. Gehler, M. Pato, and G. Bertone, J. Cosmol. Astropart. Phys. 09 (2015) 048.

[103] K. K. Boddy and J. Kumar, Phys. Rev. D 92, 023533 (2015).

[104] K. Abazajian, G. M. Fuller, and W. H. Tucker, Astrophys. J. 562, 593 (2001).

[105] A. Boyarsky, A. Neronov, O. Ruchayskiy, M. Shaposhnikov, and I. Tkachev, Phys. Rev. Lett. 97, 261302 (2006).

[106] C. R. Watson, J. F. Beacom, H. Yuksel, and T. P. Walker, Phys. Rev. D 74, 033009 (2006).

[107] K. N. Abazajian, M. Markevitch, S. M. Koushiappas, and R. C. Hickox, Phys. Rev. D 75, 063511 (2007).

[108] K. Abazajian and S. M. Koushiappas, Phys. Rev. D 74, 023527 (2006).

[109] A. R. Pullen, R.-R. Chary, and M. Kamionkowski, Phys. Rev. D 76, 063006 (2007). 
[110] H. Yuksel, J. F. Beacom, and C. R. Watson, Phys. Rev. Lett. 101, 121301 (2008).

[111] A. Boyarsky, D. Iakubovskyi, O. Ruchayskiy, and V. Savchenko, Mon. Not. R. Astron. Soc. 387, 1361 (2008).

[112] A. Boyarsky, D. Malyshev, A. Neronov, and O. Ruchayskiy, Mon. Not. R. Astron. Soc. 387, 1345 (2008).

[113] M. Loewenstein, A. Kusenko, and P. L. Biermann, Astrophys. J. 700, 426 (2009).

[114] A. Boyarsky, O. Ruchayskiy, and M. Shaposhnikov, Annu. Rev. Nucl. Part. Sci. 59, 191 (2009).

[115] K. N. Abazajian, P. Agrawal, Z. Chacko, and C. Kilic, Phys. Rev. D 85, 123543 (2012).

[116] M. Loewenstein and A. Kusenko, Astrophys. J. 751, 82 (2012).

[117] C. Jackson, G. Servant, G. Shaughnessy, T. M. Tait, and M. Taoso, J. Cosmol. Astropart. Phys. 07 (2013) 021.

[118] S. Horiuchi, P. J. Humphrey, J. Oñorbe, K. N. Abazajian, M. Kaplinghat, and S. Garrison-Kimmel, Phys. Rev. D 89, 025017 (2014).

[119] K. K. Boddy, J. L. Feng, M. Kaplinghat, Y. Shadmi, and T. M. P. Tait, Phys. Rev. D 90, 095016 (2014).

[120] K. C. Y. Ng, S. Horiuchi, J. M. Gaskins, M. Smith, and R. Preece, Phys. Rev. D 92, 043503 (2015).

[121] E. Figueroa-Feliciano et al., arXiv:1506.05519.

[122] S. Riemer-Sorensen et al., Astrophys. J. 810, 48 (2015).

[123] K. Nandra et al., arXiv:1306.2307.

[124] S. Zhang et al. (HERD Collaboration), Proc. SPIE Int. Soc. Opt. Eng. 9144, 91440X (2014).
[125] M. Srednicki, S. Theisen, and J. Silk, Phys. Rev. Lett. 56, 263 (1986).

[126] L. Bergström and H. Snellman, Phys. Rev. D 37, 3737 (1988).

[127] A. Bouquet, P. Salati, and J. Silk, Phys. Rev. D 40, 3168 (1989).

[128] L. Bergstrom, P. Ullio, and J. H. Buckley, Astropart. Phys. 9, 137 (1998).

[129] J. Hisano, S. Matsumoto, and M. M. Nojiri, Phys. Rev. D 67, 075014 (2003).

[130] M. Gustafsson, E. Lundstrom, L. Bergstrom, and J. Edsjo, Phys. Rev. Lett. 99, 041301 (2007).

[131] G. D. Mack, T. D. Jacques, J. F. Beacom, N. F. Bell, and H. Yuksel, Phys. Rev. D 78, 063542 (2008).

[132] G. Bertone, C. B. Jackson, G. Shaughnessy, T. M. P. Tait, and A. Vallinotto, Phys. Rev. 80, 023512 (2009).

[133] R. Essig, E. Kuflik, S. D. McDermott, T. Volansky, and K. M. Zurek, J. High Energy Phys. 11 (2013) 193.

[134] K. C. Y. Ng, R. Laha, S. Campbell, S. Horiuchi, B. Dasgupta, K. Murase, and J.F. Beacom, Phys. Rev. D 89, 083001 (2014).

[135] A. Albert, G. A. Gómez-Vargas, M. Grefe, C. Muñoz, C. Weniger, E. D. Bloom, E. Charles, M. N. Mazziotta, and A. Morselli, J. Cosmol. Astropart. Phys. 10 (2014) 023.

[136] M. Ackermann et al. (Fermi-LAT Collaboration), Phys. Rev. D 91, 122002 (2015). 